\title{
Expression of early growth response gene-1 in precancerous lesions of gastric cancer
}

\author{
SEON-YOUNG PARK ${ }^{1}$, JI-YOUNG KIM $^{1}$, SU-MI LEE ${ }^{1}$, JIN OOK CHUNG ${ }^{2}$, \\ KYUNG-HWA LEE ${ }^{3}$, CHUNG-HWAN JUN ${ }^{1}$, CHANG-HWAN PARK ${ }^{1}$, HYUN-SOO KIM ${ }^{1}$, \\ SUNG-KYU CHOI ${ }^{1}$, JONG-SUN REW ${ }^{1}$, YOUNG-DO JUNG ${ }^{4}$ and YONG HAN LEE ${ }^{5}$
}

\begin{abstract}
Department of ${ }^{1}$ Gastroenterology and Hepatology; ${ }^{2}$ Department of Endocrinology; ${ }^{3}$ Department of Pathology, Chonnam National University Research Institute of Medical Sciences; ${ }^{4}$ Department of Biomedical Science and Technology,

Chonnam National University Medical School, Gwangju 501-757; ${ }^{5}$ Department of Biological Sciences,

College of Biological Science and Biotechnology, Konkuk University, Seoul 143-701, Republic of Korea
\end{abstract}

Received May 3, 2015; Accepted June 2, 2016

DOI: $10.3892 / \mathrm{ol} .2016 .4962$

\begin{abstract}
Several studies have demonstrated a correlation between the expression of early growth response gene-1 (EGR-1) and the progression of gastric cancers at advanced stages. However, the effects of EGR-1 expression on human gastric cancer progression, particularly on precancerous lesions, have not been investigated. In this study, we evaluate EGR-1 expression levels in target mucosa from patients with early gastric cancer and precancerous lesions, and assess whether EGR-1 expression affects the oncogenic phenotypes of human gastric cancer cells. EGR-1 protein levels were measured in tissues from subjects with normal mucosa $(n=6)$, low-grade dysplasia $(n=6)$, high-grade dysplasia $(n=4)$ and adenocarcinoma $(n=3)$ using enzyme-linked immunosorbent assay and immunohistochemistry analyses. We also investigated the role of EGR-1 in tumor cell behavior by transiently expressing a dominant active EGR-1 variant in cultured cells. A positive correlation was observed between EGR-1 expression and gastric carcinogenesis $(\mathrm{P}=0.016)$. Furthermore, there was an increase in nuclear and cytoplasmic expression of EGR-1 in accordance with the histological grade ( $\mathrm{P}$ for trends $=0.003$ and 0.003 , respectively), and a positive association between the sum of the nuclear and cytoplasmic EGR-1 expression values and the histological grade $(\mathrm{P}=0.003)$. In addition, transient overexpression of EGR-1 enhanced cell proliferation, stimulated cell migration, and promoted the phosphorylation of p38 MAPK and AKT in gastric cancer cells in vitro. Our findings demonstrate that EGR-1 may contribute to the early stages of gastric carcinogenesis via the alteration of tumor cell behaviors.
\end{abstract}

Correspondence to: Professor Jong-Sun Rew, Department of Gastroenterology and Hepatology, Chonnam National University Medical School, 42 Jaebongro, Dong-gu, Gwangju 501-757, Republic of Korea

E-mail: drpsy@jnu.ac.kr

Key words: early growth response gene-1, gastric cancer, dysplasia

\section{Introduction}

Gastric cancer is one of the most common types of cancer worldwide, particularly in East Asian populations. However, while the precise mechanism that underlies gastric carcinogenesis is not fully understood, multiple genetic alterations in tumor suppression genes, oncogenes, cell adhesion molecules, the growth factor/receptor system and DNA repair genes have been observed to be involved in this process. Among the molecular markers that exhibit variable expression levels, the expression of early growth response-1 (EGR-1), which is considered a tumor suppressor gene in a number of cell types, was observed to be elevated in gastric cancer tissues (1). EGR-1 is functionally implicated in numerous critical biological processes, including inflammation, cell proliferation, differentiation, vascular wound response and cancer progression (2). Furthermore, it has been suggested that EGR-1 may play a significant role in carcinogenesis and cancer progression in the stomach via the alteration of tumor cell behaviors, including migration and invasion (3). However, the effects of EGR-1 expression on human gastric cancer progression, particularly in precancerous lesions, have yet to be investigated. The aims of this study were to measure the expression levels of EGR-1 in the target mucosa of patients with early gastric cancer and precancerous lesions, and to evaluate whether EGR-1 affects oncogenic phenotypes of human gastric cancer cells.

\section{Materials and methods}

Patients and specimens. The expression levels of EGR-1 protein were evaluated in 19 gastric tissues harvested from patients with chronic gastritis $(n=6)$, low-grade dysplasia (LGD; $n=6)$, high-grade dysplasia (HGD; $n=4$ ) or adenocarcinoma (ADC; $\mathrm{n}=3$ ) by endoscopic biopsy. All patients had undergone endoscopic mucosal resection or endoscopic submucosal dissection for known dysplasia or T1 ADC at Chonnam National University Hospital, Korea, between July and December 2014. All resected specimens were evaluated by histological examination on the basis of the Vienna classification system (4). 
Patients were considered to be infected with Helicobacter pylori if the results of at least one of three diagnostic tests (rapid urease test, histology results and $\left[{ }^{13} \mathrm{C}\right]$-urea breath test) were positive. All specimens were collected with the informed consent of patients, and the study was approved by the ethics committee of Chonnam National University Hospital (IRB no. CNUH-2014-144).

Cell culture and transfection. Human gastric carcinoma AGS cells were purchased from the American Type Culture Collection (Manassas, VA, USA). Cells were cultured at $37^{\circ} \mathrm{C}$ in a $5 \%$ $\mathrm{CO}_{2}$ atmosphere with Dulbecco's modified Eagle's medium (Gibco; Thermo Fisher Scientific, Inc., Waltham, MA, USA) supplemented with $10 \%$ heat-inactivated fetal bovine serum, $1 \%$ penicillin-streptomycin and $0.2 \%$ gentamycin $(50 \mathrm{mg} / \mathrm{ml}$ solution; Gibco; Thermo Fisher Scientific, Inc.). For transfection studies, cells were seeded on six-well plates and incubated overnight. Adhered cells were then transiently transfected with the pcDNA3.1/EGR-1 (I293F) expression vector (provided by Professor Yong Han Lee), which expresses a dominant active form of EGR-1 [(DA)-EGR-1].

MTT assay. Cell viability was evaluated using the 3-(4,5dimethylthiazole-2-yl)-2,5-diphenyl tetrazolium bromide (MTT) assay. Briefly, AGS cells were seeded in 96-well plates and allowed to adhere overnight at $37^{\circ} \mathrm{C}$. Following incubation, $20 \mu \mathrm{l}$ MTT solution was added to each well and the plates were incubated for $4 \mathrm{~h}$ at $37^{\circ} \mathrm{C}$ with $5 \% \mathrm{CO}_{2}$. The MTT solution was then removed, and $200 \mu \mathrm{l}$ dimethyl sulfoxide was added to each well. Viability was quantified by measuring the optical density of each well at a wavelength of $550 \mathrm{~nm}\left(\mathrm{OD}_{550}\right)$.

Cell migration assay. To assess the effects of EGR-1 expression on cell migration, cell migration assays were performed in six-well plates (Corning ${ }^{\circledR} 3516$ Costar $^{\circledR}$ six-well x $16.8 \mathrm{ml}$ flat bottom cell culture microplate; Corning Life Sciences, Tewksbury, MA, USA). To create wound gaps, AGS cells were seeded on the culture plate inserts, and gently removed using sterile tweezers after $0,6,12,24$ and $48 \mathrm{~h}$ of incubation. The progress of wound closure was monitored and photographed using an inverted microscope. Wound size was measured at six different positions on the photographs, and the average wound size at each position was calculated.

Evaluation of EGR-1 expression. The EGR-1 protein expression levels were measured within tissues from patients with normal mucosa $(n=6)$, LGD $(n=6)$, HGD $(n=4)$ or ADC $(n=4)$ using a human enzyme-linked immunosorbent assay (ELISA) kit (Cloud-Clone Corp., Houston, TX, USA), according to the manufacturer's instructions. The detection limit of the assay for EGR-1 was $0.1 \mathrm{ng} / \mathrm{ml}$. All measurements were performed in duplicate.

For immunohistochemical staining, 4- $\mu$ m-thick sections were generated from the 19 gastric tissues from subjects with normal mucosa, LGD, HGD or ADC. The tissue sections were then deparaffinized, rehydrated and retrieved with retrieval buffer. Tissues were treated with peroxidase-blocking solution (Dako, Glostrup, Denmark) to block endogenous peroxidase activity, and incubated at $4^{\circ} \mathrm{C}$ overnight with polyclonal rabbit anti-human EGR-1 antibody (diluted 1:100). After washing with Tris-buffered saline containing Tween-20 (TBST), tissues were stained using the Dako Real $^{\mathrm{TM}}$ Envision HRP/DAB detection system (Dako). Stained tissues were viewed and photographed using a light microscope. The immunoreactivity of each sample (intensity, total area and pattern of immunostaining) was evaluated independently by two observers who had no knowledge of the clinical outcomes. In cases where there was a discrepancy, a consensus was reached after further evaluation. The staining intensity was graded using the following numerical scale: 0 , no staining of cancer cells; 1 , weak staining; 2 , moderate staining; and 3, strong staining. Specifically, we analyzed the staining intensity within the nucleus and cytoplasm, respectively. The overall scores were calculated as the sum of the staining intensity in the nuclei and the cytoplasm. As such, these scores could range from 0 to 6 . Specimens with a score $>3$ were regarded as EGR-1 expression-positive, while those with a score $\leq 3$ were considered EGR-1 expression-negative.

Western blot analysis. Cells were lysed in RIPA buffer (1 M Tris- $\mathrm{HCl}, 150 \mathrm{mM} \mathrm{NaCl}, 1 \%$ Triton X-100 and $2 \mathrm{mM}$ EDTA) containing a phosphatase inhibitor and a protease inhibitor cocktail (Sigma-Aldrich, St. Louis, MO, USA). The cell lysates (20 $\mu \mathrm{g}$ protein) were then separated by $10 \%$ sodium dodecyl sulphate-polyacrylamide gel electrophoresis and electrophoretically transferred to polyvinylidene fluoride membranes. After blocking with 5\% skimmed milk in TBST for $1 \mathrm{~h}$, membranes were blotted with EGR-1- or $\beta$-actin-specific antibodies (Cell Signaling Technology, Danvers, MA, USA) at room temperature for $3 \mathrm{~h}$. After washing three times with TBST, membranes were incubated with horse-radish peroxidase (HRP)-conjugated anti-rabbit immunoglobulin secondary antibodies (1:2,000 dilution; Cell Signaling Technology) at room temperature for $1 \mathrm{~h}$. After three washes with TBST, membranes were incubated with enhanced chemiluminescence reagent, and protein bands were visualized using a Fuji LAS-3000 image analyzer (Fuji Film, Tokyo, Japan).

Statistical analyses. Differences in EGR-1 protein levels and in the intensity values obtained from the EGR-1 immunohistochemical staining analyses were evaluated using a non-parametric Kruskal-Wallis test. Chi-square tests (linear by linear association) were used to compare the nuclear and cytoplasmic expression of EGR-1 according to the histological grades. Associations between EGR-1 expression and clinical factors were evaluated by Chi-square tests and Mann-Whitney $\mathrm{U}$ tests. The results of the cell viability (MTT) and migration assays were assessed using two-tailed Student's t-tests. $\mathrm{P}<0.05$ was considered to indicate a statistically significant difference. All analyses were conducted using Statistical Package for Social Sciences (SPSS/PC) 20.0 software (SPSS Statistics Inc., Chicago, IL, USA).

\section{Results}

Expression of EGR-1 protein is associated with gastric carcinogenesis. We first analyzed the EGR-1 protein expression levels in gastric precancerous lesions and cancer tissues using an EGR-1-specific ELISA. A total of 19 patients 
Table I. Baseline demographic data for the patients enrolled in study.

\begin{tabular}{lccc}
\hline & $\begin{array}{c}\text { Median } \\
\text { age (range) }\end{array}$ & $\begin{array}{c}\text { Gender } \\
(\mathrm{M}: \mathrm{F})\end{array}$ & $\begin{array}{c}\text { Positive for } \\
\text { H.pylori }(\%)\end{array}$ \\
\hline Chronic gastritis (n=6) & $39(28-71)$ & $3: 3$ & $3(50)$ \\
LGD (n=6) & $67(54-76)$ & $4: 2$ & $3(50)$ \\
HGD (n=4) & $72(48-80)$ & $1: 3$ & $0(0)$ \\
T1 ADC (n=3) & $59(44-68)$ & $2: 1$ & $3(100)$ \\
\hline
\end{tabular}

H. pylori, Helicobacter pylori; LGD, low-grade dysplasia; HGD, high-grade dysplasia; T1 ADC, T1 adenocarcinoma.

Table II. Correlation between EGR-1 expression and clinicopathological features.

\begin{tabular}{|c|c|c|c|}
\hline & $\begin{array}{c}\text { EGR-1 } \\
n=6\end{array}$ & $\begin{array}{c}\text { EGR-1 }{ }^{+} \\
\mathrm{n}=13\end{array}$ & P-value \\
\hline Age $($ mean $\pm S D)$ & $45.3 \pm 19.6$ & $64.6 \pm 10.8$ & 0.062 \\
\hline Gender (M:F) & $3: 3$ & $7: 6$ & 0.999 \\
\hline Positive H.pylori & $3(50.0 \%)$ & $6(46.2 \%)$ & 0.999 \\
\hline Location of specimen & & & 0.459 \\
\hline Lower $1 / 3$ & $5(83.3 \%)$ & $7(53.8 \%)$ & \\
\hline Mid 1/3 & $0(0 \%)$ & $4(30.8 \%)$ & \\
\hline Upper $1 / 3$ & $1(16.7 \%)$ & $2(15.4 \%)$ & \\
\hline Pathology & & & 0.001 \\
\hline Chronic gastritis & $6(100 \%)$ & $0(0 \%)$ & \\
\hline LGD & $0(0 \%)$ & $6(46.2 \%)$ & \\
\hline HGD & $0(0 \%)$ & $4(30.8 \%)$ & \\
\hline T1 ADC & $0(0 \%)$ & $3(23.1 \%)$ & \\
\hline
\end{tabular}

EGR-1, early growth response gene-1; SD, standard deviation; H. pylori, Helicobacter pylori; LGD, low-grade dysplasia; HGD, high-grade dysplasia; T1 ADC, T1 adenocarcinoma.

(median age, 65 years; 10 males) were included in this study. The baseline demographic data of the patients are summarized in Table I. Nine (47.4\%) of the 19 patients were infected with $H$. pylori. The results of these assays indicated that there was a positive correlation between EGR-1 expression and the histological grade $(\mathrm{P}=0.016$, Fig. 1A). In addition, we assessed EGR-1 expression by immunohistochemical staining of formalin-fixed, paraffin-embedded tissue blocks obtained from the 19 enrolled patients (Fig. 1B). Nuclear and cytoplasmic expression of EGR-1 increased according to the histological grade ( $\mathrm{P}$ for trends $=0.003$ and 0.003 , respectively), and there was a positive association between the sum of the nuclear and cytoplasmic EGR-1 expression levels and the histological grade $(\mathrm{P}=0.003$, Fig. $1 \mathrm{C})$. When the expression of EGR-1 was classified as positive or negative, according to the immunohistochemistry score, there was a statistically significant association between the positive expression of EGR-1 and the histological grade ( $\mathrm{P}$ for trend=0.001). Conversely, gender,
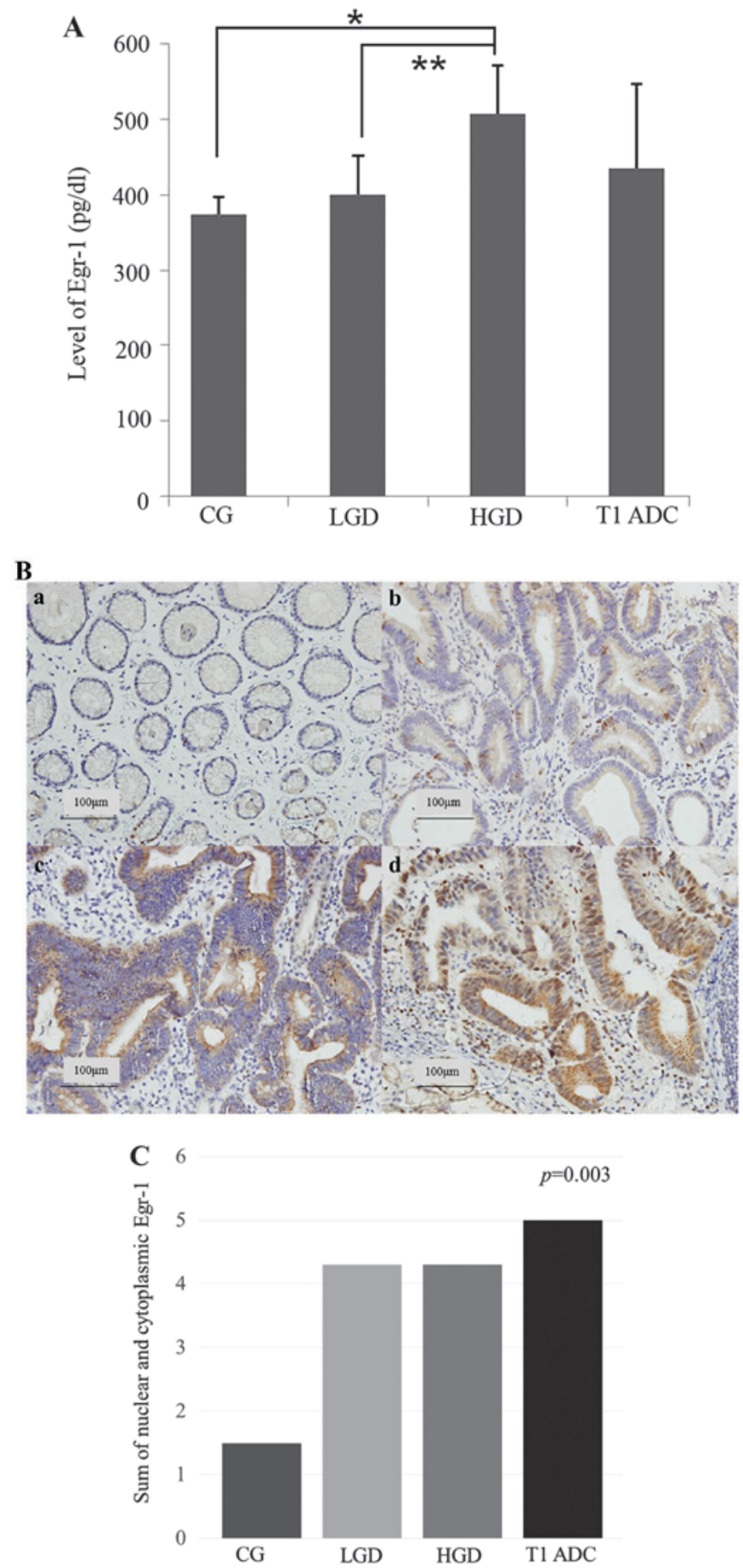

Figure 1. Expression of early growth response gene-1 (EGR-1) in precancerous lesions and gastric cancer. (A) There was a positive correlation between EGR-1 protein levels (enzyme-linked immunosorbent assay) and the histological grade $(\mathrm{P}=0.016)$. (B) EGR-1 expression by immunohistochemical staining of tissue blocks obtained from 19 enrolled patients. a, chronic gastritis (CG); b, low-grade dysplasia (LGD); c, high-grade dysplasia (HGD); d, T1 adenocarcima (ADC) (original magnification, x200). (C) Nuclear and cytoplasmic expression of EGR-1 was increased according to histological grade. (C) EGR-1 is positively associated with histological grade; ${ }^{*} \mathrm{P}<0.005$ and ${ }^{* *} \mathrm{P}<0.05$.

age, presence of $H$. pylori and tumor location were not associated with EGR-1 expression (Table II).

EGR-1 overexpression promotes cell proliferation in gastric cancer cells. MTT assays were used to assess the potential 


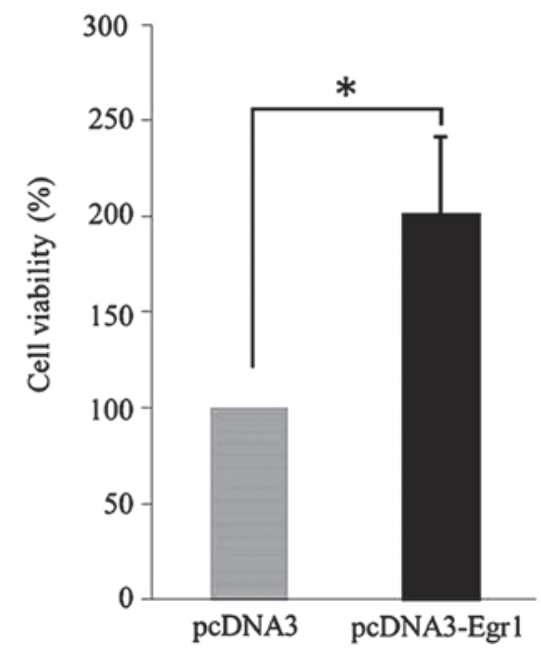

Figure 2. Effects of early growth response gene-1 (EGR-1) on the proliferation of AGS cells. AGS cells were transfected with pcDNA3 or pcDNA3/EGR-1 plasmid. EGR-1 upregulation promoted the proliferation of gastric cancer cells. The viable cell number was determined by 3-(4,5-dimethylthiazole-2-yl)-2,5-diphenyl tetrazolium bromide colorimetric assay and indicated as a percentage of the empty vector. The result represents the average of three independent experiments. ${ }^{*} \mathrm{P}<0.05$.

effects of EGR-1 expression on the proliferation of human gastric cancer cells. Compared with cells transfected with the empty vector, AGS cells transfected with the pcDNA3.1/EGR-1 (I293F) expression vector, which encodes (DA)-EGR-1, exhibited enhanced rates of proliferation ( $\mathrm{P}=0.021$, Fig. 2$)$.

Overexpression of EGR-1 promotes the migration of gastric cancer cells. We examined whether EGR-1 expression promotes the migration of gastric cancer cells using a wound-healing assay. Compared with the cells transfected with the empty vector, AGS cells transfected with the EGR-1 expression vector exhibited significant reductions in wound sizes (Fig. 3A). Furthermore, quantitative analyses revealed that overexpression of EGR-1 stimulated gastric cancer cell migration (Fig. 3B).

EGR-1 expression promotes the phosphorylation of $p 38$ $M A P K$ and $A K T$. While transfection of AGS cells with the EGR-1 expression vector resulted in increased levels of AKT and p38 MAPK phosphorylation, this EGR-1 overexpression had no effect on the total expression levels of AKT and MAPK (Fig. 4). Therefore, these findings indicate that EGR-1 promotes the activation of $\mathrm{p} 38$ MAPK and AKT.

\section{Discussion}

The principal findings of this study were as follows: i) expression of EGR-1 protein, as detected by ELISA, was associated with gastric carcinogenesis; ii) there was an increase in the nuclear and cytoplasmic levels of EGR-1 expression in accordance with the histological grades; iii) EGR-1 overexpression promoted cell proliferation and migration in gastric cancer cells in vitro; and iv) overexpression of EGR-1 resulted in increased phosphorylation of $\mathrm{p} 38$ MAPK and AKT in vitro.

EGR-1 functions as either a growth-promoting factor or as a tumor suppressor (5-8), and previous studies demonstrated
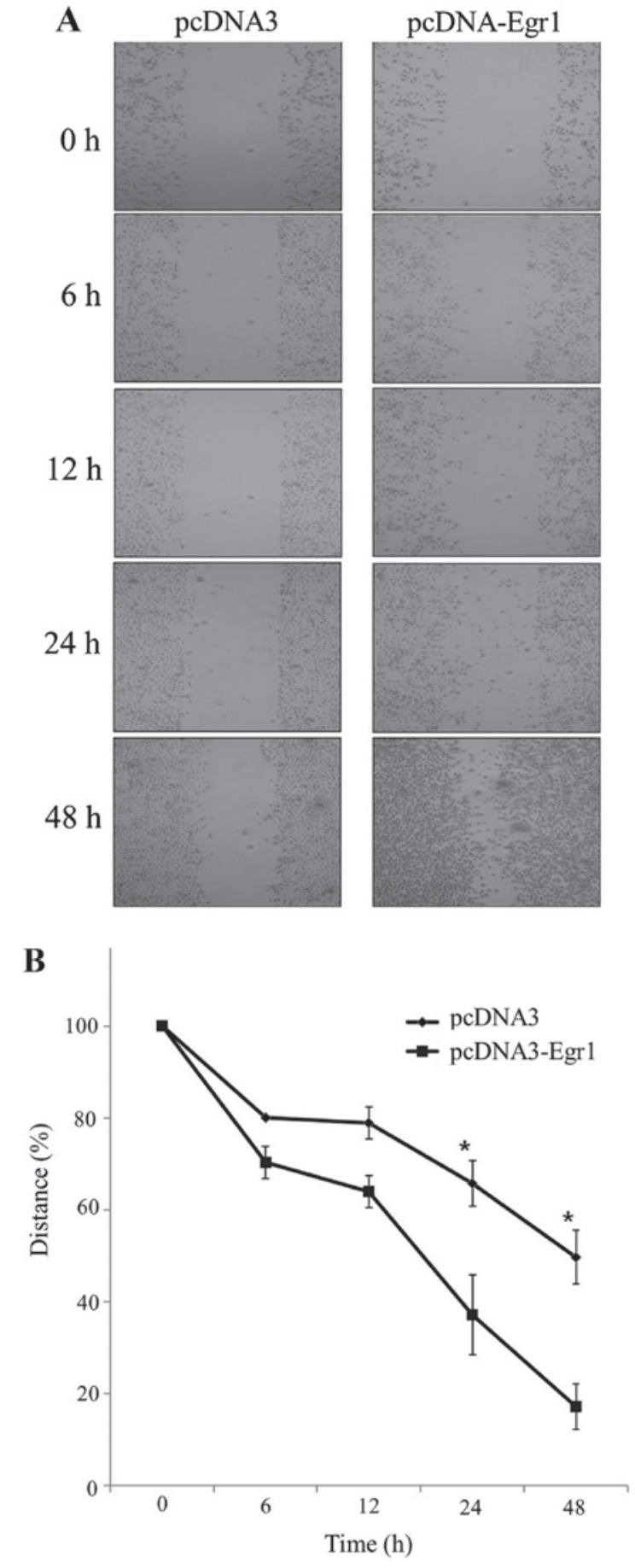

Figure 3. Effects of early growth response gene-1 (EGR-1) on the migration of gastric cancer cells. (A) Wound healing assay using EGR-1 transfected cells was performed, and (B) graphs of cell migration are displayed as relative healing distances (mean \pm standard error; ${ }^{*} \mathrm{P}<0.005$ ). Compared with empty vector transfection cells, EGR-1-transfected cells significantly reduced the wound sizes after EGR-1 was plasmid-transfected.

a positive correlation between the expression of EGR-1 and gastric cancer progression during the advanced stages $(3,9)$. The expression of EGR-1 is rapidly induced by a number of extracellular stimuli, including growth factors, cytokines, hypoxia, injury-related stimuli and bacterial toxins $(2,10)$. Keates et al demonstrated that exposure to $c a g+H$. pylori promoted $E G R-1$ gene expression in gastric epithelial cells, and that this upregulation may contribute to cancer 


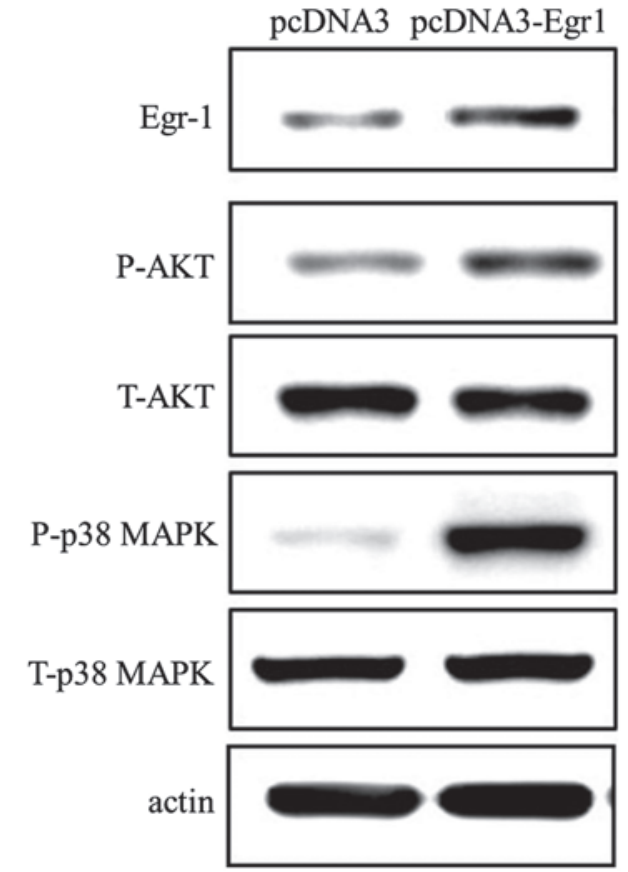

Figure 4. Effects of early growth response gene-1 (EGR-1) on p38 MAPK and AKT. In AGS cells, EGR-1 transfection stimulated the phosphorylation of AKT and $\mathrm{p} 38 \mathrm{MAPK}$.

pathogenesis (11). Notably, gastric carcinogenesis is a multifactorial process for which $H$. pylori infection is the most significant risk factor (12). Since constant exposure of the gastric epithelium to $H$. pylori may cause frequent cellular activation and upregulation of EGR-1, we speculated that EGR-1 expression may play a role in the progression of gastric cancer, not only during advanced stages (including invasion or distant metastasis), but also during early stages (including the dysplasia-carcinoma carcinogenic pathway). Gastric cancers are classified histologically into two main types: intestinal and diffuse. Intestinal-type gastric carcinomas are considered to be derived from gastric mucosal cells. These carcinomas are also histologically differentiated based on well-defined glandular structures that exhibit an expanding growth pattern and which develop through well-characterized sequential stages, including chronic gastritis, atrophy, intestinal metaplasia and dysplasia, which is an atypical change in the epithelium that is considered to be precancerous (13). Moreover, there is abundant evidence suggesting that most gastric cancers, particularly the intestinal subtype, develop via an intestinal metaplasia-dysplasia-carcinoma carcinogenic pathway (14).

Currently, there are no published studies that have examined the correlation between EGR-1 expression and precancerous gastric lesions. A previous study, however, detected high levels of EGR-1 mRNA and protein expression in precancerous lesions of the esophagus (10). Likewise, in this study, we observed that EGR-1 protein expression levels were elevated in precancerous stomach lesions (LGD and HGD). Furthermore, compared with tissues obtained from healthy patients, we observed an increase in the nuclear and cytoplasmic expression of EGR-1 in gastric tissues harvested from patients with precancerous lesions by immunohistochemistry. These findings therefore indicate that EGR-1 may be involved in the progression of intestinal-type gastric carcinomas at stages as early as the formation of precancerous lesions, including dysplasia.

To examine the functional significance of EGR-1 in human gastric cancer cells, we transfected a gastric cancer cell line with either an EGR-1 overexpression vector or a control vector. A previous study demonstrated that EGR-1 expression resulted in increased tumor cell migration and invasion by promoting $\beta$-catenin expression in human gastric cancer cells (9). In addition, Myung et al demonstrated that a knockdown of EGR-1 expression in human gastric cancer cell lines resulted in decreased rates of tumor cell invasion and migration (3). Consistent with these findings, our results indicate that EGR-1 overexpression promotes cell proliferation and migration in gastric cancer cells. Additionally, they demonstrate that EGR-1 expression promotes the phosphorylation of p38 MAPK and AKT. Thus, EGR-1 activation due to exogenous stimuli, including $H$. pylori infection or a high salt diet, may contribute to gastric epithelial cell hyperproliferation leading to gastric dysplasia. Based on the American Joint Committee on Cancer and the International Union Against Cancer (AJCC/UICC) staging, T1 gastric cancers include tumors that invade the lamina propria, muscularis mucosae or submucosa (15), and are defined as unequivocal neoplastic epithelia that are confined to the basement membrane. Dysplasia is categorized as either low or high grade, depending on the degree of cytological and architectural atypia of the pits and surface epithelium $(16,17)$. Therefore, EGR-1 activation may contribute to the migration of neoplastic cells, leading to the progression of dysplasia to $\mathrm{T} 1$ gastric cancer.

In conclusion, our findings demonstrate that EGR-1 may be involved in the early stages, as well as the latter stages, of gastric carcinogenesis through the alteration of tumor cell behaviors.

\section{Acknowledgements}

This study was supported by a grant from Chonnam National University Hospital Biomedical Research Institute (CRI14014-1).

\section{References}

1. Kobayashi D, Yamada M, Kamagata C, Kaneko R, Tsuji N, Nakamura M, Yagihashi A and Watanabe N: Overexpression of early growth response-1 as a metastasis-regulatory factor in gastric cancer. Anticancer Res 22: 3963-3970, 2002.

2. Thiel G and Cibelli G: Regulation of life and death by the zinc finger transcription factor EGR-1. J Cell Physiol 193: 287-292, 2002.

3. Myung E, Park YL, Kim N, Chung CY, Park HB, Park HC, Myung DS, Kim JS, Cho SB, Lee WS and Joo YE: Expression of early growth response-1 in human gastric cancer and its relationship with tumor cell behaviors and prognosis. Pathol Res Pract 209: 692-699, 2013.

4. Schlemper RJ, Riddell RH, Kato Y, Borchard F, Cooper HS, Dawsey SM, Dixon MF, Fenoglio-Preiser CM, Fléjou JF, Geboes K, et al: The vienna classification of gastrointestinal epithelial neoplasia. Gut 47: 251-255, 2000.

5. de Belle I, Huang RP, Fan Y, Liu C, Mercola D and Adamson ED: p53 and Egr-1 additively suppress transformed growth in ht1080 cells but Egr-1 counteracts p53-dependent apoptosis. Oncogene 18: 3633-3642, 1999

6. Yu J, Zhang SS, Saito K, Williams S, Arimura Y, Ma Y, Ke Y, Baron V, Mercola D, Feng GS, et al: Pten regulation by AKT-EGR1-ARF-PTEN axis. EMBO J 28: 21-33, 2009. 
7. Kim J, Lee YH, Kwon TK, Chang JS, Chung KC and Min DS: Phospholipase D prevents etoposide-induced apoptosis by inhibiting the expression of early growth response- 1 and phosphatase and tensin homologue deleted on chromosome 10 . Cancer Res 66: 784-793, 2006.

8. Mahalingam D, Natoni A, Keane M, Samali A and Szegezdi E: Early growth response-1 is a regulator of DR5-induced apoptosis in colon cancer cells. Br J Cancer 102: 754-764, 2010.

9. Sun T, Tian H, Feng YG, Zhu YQ and Zhang WQ: Egr-1 promotes cell proliferation and invasion by increasing $\beta$-catenin expression in gastric cancer. Dig Dis Sci 58: 423-430, 2013.

10. Wu MY, Liang YR, Wu XY and Zhuang CX: Relationship between Egr-1 gene expression and apoptosis in esophageal carcinoma and precancerous lesions. World J Gastroenterol 8: 971-975, 2002

11. Keates S, Keates AC, Nath S, Peek RM Jr and Kelly CP: Transactivation of the epidermal growth factor receptor by cag+ helicobacter pylori induces upregulation of the early growth response gene Egr-1 in gastric epithelial cells. Gut 54: 1363-1369, 2005.
12. Conteduca V, Sansonno D, Lauletta G, Russi S, Ingravallo G and Dammacco F: H. pylori infection and gastric cancer: state of the art (review). Int J Oncol 42: 5-18, 2013.

13. Yuasa Y: Control of gut differentiation and intestinal-type gastric carcinogenesis. Nat Rev Cancer 3: 592-600, 2003.

14. Correa P: Human gastric carcinogenesis: a multistep and multifactorial process-first American Cancer Society award lecture on cancer epidemiology and prevention. Cancer Res 52: 6735-6740, 1992.

15. Edge SB and Compton CC: The American joint committee on cancer: The 7th edition of the AJCC cancer staging manual and the future of TNM. Ann Surg Oncol 17: 1471-1474, 2010.

16. Rugge M, Correa P, Dixon MF, Hattori T, Leandro G, Lewin K, Riddell RH, Sipponen P and Watanabe H: Gastric dysplasia: the Padova international classification. Am J Surg Pathol 24: 167-176, 2000.

17. Stolte M: The new vienna classification of epithelial neoplasia of the gastrointestinal tract: advantages and disadvantages. Virchows Arch 442: 99-106, 2003. 